\title{
Impacts of a gross anatomy laboratory course on medical students' emotional reactions in Taiwan: the role of high-level emotions
}

\author{
Ruei-Jen Chiou', Po-Fang Tsai ${ }^{1}$ and Der-Yan $\operatorname{Han}^{3 *}$
}

\begin{abstract}
Background: Gross anatomy laboratory course at medical school is usually an important learning subject for medical students; however, seeing a cadaver often makes them feel uncomfortable. According to the broaden-andbuild theory, positive emotions broaden our inventory of thoughts and actions, and build physical, mental, and social resources. Research on positive psychology found that through direct thanks and positive reframing, people who feel gratitude show fewer depressive symptoms. The present study tried to reduce students' negative emotions towards cadavers by sequential activities, such as family interviews and an initiation ceremony, which induced gratitude and other positive emotions.

Methods: The Emotional Reactions Towards Cadavers Scale (ERTCS) was used to evaluate medical students' emotional reactions after they see a cadaver. Third year medical students $(n=105)$ at Taipei Medical University in northern Taiwan completed ERTCS on three occasions within a single semester during academic year 2016. Repeated-measures ANOVA and hierarchical regression analyses were then conducted to identify any changes in the emotional reactions of these students.

Results: The ERTCS showed satisfactory internal consistency and a three-factor structure, i.e., negative emotions, high-level emotions, and excited emotions. High-level emotions were the highest, and negative emotions were the lowest among the three in our sample. Three-wave data showed that participants' high-level emotions increased, negative emotions decreased, and the former simultaneously predicted the latter after controlling for the influence of gender, religious beliefs, experience of the death of a family member or friend, and burnout level.

Conclusions: While past research usually focused on coping strategies to reduce medical students' negative emotions, our study supported the broaden-and-build theory, which emphasizes positive emotions, and demonstrated that elevating medical students' gratitude to 'silent mentors' is an effective way. It is suggested that combining dissection courses with medical humanities can help students successfully handle negative emotions during a gross anatomy laboratory course.
\end{abstract}

Keywords: Death anxiety, High-level emotion, Ceremony, Silent mentor, Gross anatomy education

* Correspondence: handeyan@tmu.edu.tw

${ }^{3}$ Section of Liberal Arts, Center for General Education, Taipei Medical

University, 250 Wu-Xing St., Xinyi District, 11031 Taipei, Taiwan

Full list of author information is available at the end of the article

(c) The Author(s). 2021 Open Access This article is licensed under a Creative Commons Attribution 4.0 International License, which permits use, sharing, adaptation, distribution and reproduction in any medium or format, as long as you give appropriate credit to the original author(s) and the source, provide a link to the Creative Commons licence, and indicate if changes were made. The images or other third party material in this article are included in the article's Creative Commons licence, unless indicated otherwise in a credit line to the material. If material is not included in the article's Creative Commons licence and your intended use is not permitted by statutory regulation or exceeds the permitted use, you will need to obtain permission directly from the copyright holder. To view a copy of this licence, visit http://creativecommons.org/licenses/by/4.0/ The Creative Commons Public Domain Dedication waiver (http://creativecommons.org/publicdomain/zero/1.0/) applies to the data made available in this article, unless otherwise stated in a credit line to the data. 
The gross anatomy laboratory course is an important learning subject for medical students, and how teachers prepare them for the emotional impacts before and during the class is a critical concern for medical education. Most young medical students have no experience with death of a close relative or a friend, so that when they go into a dissection room and see a cadaver, they usually manifest multiple physiological and psychological symptoms. Previous studies showed that medical students in a dissection room experience many physiological [1-7] and psychological problems $[1,2,5,8]$. Symptoms experienced by more than $20 \%$ of participants are listed in Table 1. Generally, about $30 \%$ of medical students' experience at least one of these adverse impacts when they take a gross anatomy laboratory course [4].

Researchers have tried to explore the reasons causing medical students to suffer so much. Bernhardt et al. used self-composed questionnaires, including some items about previous experiences with death and dying, to investigate psychological stress of 155 first-year medical students (112 females, 43 males) in Germany [3]. They found that students who had no experience with the death of a relative or friend had higher fears when facing body donors. Another study used a checklist and questionnaires to investigate the sensations or reactions of 425 students (79.6\% females, $20.4 \%$ males) to dissection, and pointed out that students who were not prepared or did not have deep thoughts about life and death during dissection had higher death anxiety [9]. Those two studies suggest that helping students face life and death issues could be a good strategy to reduce students' negative emotions when they take a gross anatomy laboratory course.

\section{Teachers' interventions and students' coping strategies}

There have been many attempts proposed by teachers to lower psychological impacts on students of seeing a cadaver. In one attempt, teachers showed students a video about anatomical information before the beginning of the course to lower students' anxiety level [10-12]. Another intervention provided senior students' help with first-year students, and found that fewer of them showed anxiety, light-headedness, headaches, and vomiting, and there were also fewer reactions of discomfort to the smell of cadavers and the dissection room [7]. Another study that guided students in groups to share their emotions and thoughts, found that most of the participating students showed that they respected the body donors because donors were living persons before, or they treated donors as if they were their future patients [13].

Table 1 Literature review of physiological and psychological problems that students report in the dissection room

\begin{tabular}{|c|c|c|c|}
\hline Symptom & Author (year) & Sample size $(n)$ & Incidence (\%) \\
\hline An impulse to leave the laboratory & Bob et al. (2014) [2] & 121 & 27.2 \\
\hline Daytime flashbacks & Sándor et al. (2015) [8] & 733 & 23.7 \\
\hline \multirow[t]{2}{*}{ Dizziness } & Getachew (2014) [1] & 206 & 24 \\
\hline & Bob et al. (2014) [2] & 121 & 42.8 \\
\hline Fainting & Bob et al. (2014) [2] & 121 & 28.2 \\
\hline Feeling sick & Bernhardt et al. (2012) [3] & 94 & 33 \\
\hline Heart palpitations & Bataineh et al. (2006) [5] & 145 & 30.3 \\
\hline Insomnia & Bob et al. (2014) [2] & 121 & 20.7 \\
\hline \multirow[t]{2}{*}{ Irritation of the eyes and throat } & Saylam and Coskunol (2005) [6] & 242 & 70.9 \\
\hline & Houwink et al. (2004) [7] & 126 & 21.4 \\
\hline \multirow[t]{2}{*}{ Loss of appetite } & Getachew (2014) [1] & 206 & 39 \\
\hline & Bob et al. (2014) [2] & 121 & 26.4 \\
\hline \multirow[t]{3}{*}{ Nausea } & Getachew (2014) [1] & 206 & 30 \\
\hline & Bob et al. (2014) [2] & 121 & 53.7 \\
\hline & Dempster (2006) [4] & 141 & 20.8 \\
\hline Often think about dissection & Sándor et al. (2015) [8] & 733 & 33.4 \\
\hline \multirow[t]{2}{*}{ Recall of a cadaver's image } & Bob et al. (2014) [2] & 121 & 57 \\
\hline & Bataineh et al. (2006) [5] & 145 & 30.9 \\
\hline \multirow[t]{2}{*}{ Sweating } & Getachew (2014) [1] & 206 & 21 \\
\hline & Bob et al. (2014) [2] & 121 & 23.1 \\
\hline Trembling & Bob et al. (2014) [2] & 121 & 33.9 \\
\hline
\end{tabular}


Students also develop their own coping strategies including rationalization, focusing on tasks, talking to and staying with peers, religious praying, inspiring themselves with the vision of being medical doctors in the future, or taking a break during dissection $[1,2,5,8,13-$ 15]. Another interesting strategy is that students may name the body donors they are dissecting. Compared to those who did not name the body donors, students who had named the body donors remembered the body donors' features better, thought of the body donors' lives when they were alive, and were more welcoming to relatives' body donation decisions. Also, more than half of participating students wanted to know the life stories of the dissection body donors [16]. All such strategies helped students overcome the unpleasantness of cadaver dissection. It seems that inspiring students' humanistic spirit can reduce the impacts of seeing a cadaver.

Emotions in the Gross Anatomy Laboratory Course

When students see a cadaver in a gross anatomy laboratory course, they often feel strong negative emotions, but also other positive emotions [4-7, 9, 13-15, 17-21]. Negative and positive emotions manifested by more than $20 \%$ of students are listed in Table 2 [4, $9-11,17,18,20]$. However, one study observed that students' high-level emotions, like respect, gratefulness, and cherishing, were aroused, and negative emotions were suppressed compared to those of Western students [22]. In Eastern cultures, we believe that introducing students to know the donors and their families can increase students' gratitude, and decrease their negative emotions. Therefore, it is worth understanding the mechanism of medical students' emotional changes in a gross anatomy laboratory course in an Eastern culture.

\section{The broaden-and-build theory}

The broaden-and-build theory in positive psychology can explain the mechanism of emotional changes. According to this theory, positive emotions broaden thought-action mechanisms, whereas negative emotions narrow them [23]. Positive emotions can cause individuals to expand their personal cognition and encourage them to have novel, varied, exploratory thoughts and actions. To elaborate, positive emotions broaden one's point of view, visions, mindsets, experiences, and knowledge of the world, and build resources like skills, physical strength, mental resilience, and social relationships. For example, chasing play of a juvenile animal not only broadens its awareness of the environment, but also builds skills for future escape from predators and hunting prey. Children's play broadens the ability to control muscles, and builds social relationship and intellectual development. These actions generate positive adaptive circulation and have lasting benefits. On the contrary, negative emotions are viewed as an adaptation when an organism faces danger; they narrow thought-actions to let an organism focus on attacking or escaping for survival. Thus, positive emotions create much more positive effects in the learning process [23-25].

Gratitude, i.e., feelings of thankfulness and appreciation when someone receives another's kindness and help, has the same broaden-and-build features as other virtues such as wisdom, knowledge, courage, humanity, justice, and temperance studied in the field of positive psychology. These feelings broaden people's action to promote the well-being of others without limitations to the person who benefited them. They also build people's social connections and friendships [23]. Previous research pointed out that positive emotions undo the effects of negative emotions [26]. Research on positive psychology found that through direct thanks and positive reframing, people who feel gratitude showed fewer depressive symptoms [27]. Another study with an experimental and control group design showed that inducing gratitude can also decrease death anxiety [28].

\section{Viewpoints of constructivism in education}

Constructivism in education is a student-centered learning approach in which the individual creates or builds his or her own understanding or knowledge by interacting with the environment [29]. In the processes of teaching and learning, students take responsibility for their own learning, and teachers are just mediators. We believe that through contacting with family members and/ or friends of body donors, students can expand their thoughts and actions, by not only understanding why body donors would sacrifice themselves to help medical students in learning dissection, but also inspiring their motivation to help patients in the future. In addition, medical students can build their own resources by learning communication skills and expressing thankful feelings. In the ceremony for the body donors, students further promise to study hard and become good doctors. Expanding students' gratitude during the time they take the gross anatomy laboratory course not only inspires other positive emotions like respect, cherishing, and humility, but also helps them spontaneously transcend the original negative emotions like fear, being spooked, and escape. From the viewpoint of constructivism, experiencing the entire process is more effective than listening to a teacher on the podium a hundred times.

Gratitude can be conceptualized on two levels: state and trait. State gratitude is an immediate effect after an event, while trait gratitude is more like a personal characteristic [30]. State gratitude might have a meaningful effect on decreasing medical students' death anxiety and related negative emotions by inducing gratitude toward the body donors. Thus, we designed family interviews 
Table 2 Literature review of negative and positive emotions that students experience in a dissection room

\begin{tabular}{|c|c|c|c|}
\hline Emotion & Author (year) & Sample size $(n)$ & Incidence (\%) \\
\hline \multicolumn{4}{|l|}{ Negative } \\
\hline \multirow[t]{6}{*}{ Anxiety } & Quince et al. (2011) [17] & 156 & 20.3 \\
\hline & Dempster (2006) [4] & 141 & 33.5 \\
\hline & Saylam and Coskunol (2005) [6] & 242 & 29.7 \\
\hline & Lamdin et al. (2012) [14] & 21 & NA \\
\hline & Bati et al. (2013) [21] & 486 & medium level \\
\hline & Houwink et al. (2004) [7] & 126 & 26 \\
\hline Apprehension & Quince et al. (2011) [17] & 156 & 37.3 \\
\hline Confusion & Quince et al. (2011) [17] & 156 & 22.9 \\
\hline \multirow[t]{3}{*}{ Fear } & Bataineh et al. (2006) [5] & 145 & 30.3 \\
\hline & Kotzé and Mole (2013) [13] & 240 & 20 \\
\hline & Tseng and Lin (2016) [15] & 12 & NA \\
\hline Uncertainty & Quince et al. (2011) [17] & 156 & 47.7 \\
\hline Being upset & Tseng and Lin (2016) [15] & 12 & NA \\
\hline \multicolumn{4}{|l|}{ Positive } \\
\hline Calmness & Arráez-Aybar et al. (2008) [9] & 425 & 33.6 \\
\hline \multirow[t]{2}{*}{ Curiosity } & Arráez-Aybar et al. (2008) [9] & 425 & 64.6 \\
\hline & Snelling et al. (2003) [20] & 364 & $4.03 \pm 0.06 / 5$ \\
\hline Enthusiasm & Quince et al. (2011) [17] & 156 & 79.7 \\
\hline \multirow[t]{3}{*}{ Being excited } & Quince et al. (2011) [17] & 156 & 76.5 \\
\hline & Qamar and Osama (2014) [19] & 60 & 34 \\
\hline & Snelling et al. (2003) [20] & 364 & $3.56 \pm 0.07 / 5$ \\
\hline Fascination & Quince et al. (2011) [17] & 156 & 83.7 \\
\hline \multirow[t]{6}{*}{ Interest } & Quince et al. (2011) [17] & 156 & 99.3 \\
\hline & Cahill and Ettarh (2009) [18] & 166 & 48.4 \\
\hline & Arráez-Aybar et al. (2008) [9] & 425 & 71.7 \\
\hline & Saylam and Coskunol (2005) [6] & 242 & 36.7 \\
\hline & Qamar and Osama (2014) [19] & 60 & 58 \\
\hline & Snelling et al. (2003) [20] & 364 & $4.26 \pm 0.06 / 5$ \\
\hline Satisfaction & Arráez-Aybar et al. (2008) [9] & 425 & 25.5 \\
\hline
\end{tabular}

and an initiation ceremony to examine the effects on students' emotions.

\section{Factors of the adverse effects}

In terms of potential factors of the aforementioned impacts, research has shown that female students reported more threatening feelings [4] and greater reluctance to cut open a cadaver [17]. When students were asked to express their feelings about dissection, male students reported more anxiety and excited feelings, whereas females reported more uncertainty and worry [17]. It was also reported that female students showed more mental distress than males did before and after they interacted with a cadaver [31]. Those studies suggested that female students have more negative physiological and psychological impacts than do male students, and take more time to recover. In terms of religious influence, a qualitative study showed that students with religious beliefs were able to endure the impacts of dissection with help from their beliefs [14]. Other research pointed out that some students pray or meditate as a coping strategy [1, 13]. It is plausible that religious beliefs can help medical students get through adverse effects of a gross anatomy laboratory course. In addition, when students feel exhausted, their emotions become more negative [32, 33]. Having experienced the death of a friend or relative is also a factor that influences students' reactions to cadavers [3]. Therefore, factors such as gender, religious 
beliefs, experience of the death of others, and one's personal exhaustion level should be controlled for to promote the accuracy when carrying out such a study.

\section{Study goals}

Based on the above inferences, the aims of the present study were to determine whether activities such as family interviews and an initiation ceremony that expand medical students' gratitude toward 'silent mentors' increase their high-level emotions and decrease their negative emotions, and whether high-level emotions predict negative emotions after controlling for variables including gender, religion, experience of the death of a family member or friend, and burnout level. To further clarify our goals, the research questions and our hypotheses are listed in Table 3. Although we matched research questions with research hypotheses, it is crucial to claim a post-positivism standpoint as the epistemological stance of this study [34, 35]. Our research design included different approaches, combining an experimental intervention with quantitative data collection. In addition, our quantitative analyses contained three different statistical techniques which were respectively deployed according to the results we sequentially attempted to establish. A post-positivist epistemology not only determined our study design, methods, and data analyses, but also influenced the way we developed our discussion, limitations, and conclusions.

\section{Materials and methods Participants}

Participants consisted of 60 male and 45 female third year medical students $(n=105)$ at Taipei Medical University in northern Taiwan. These students chose the science domain in senior high school, and entered medical university for a 6-year Doctor of Medicine (MD) degree under high expectations from their parents and teachers, and high competition with cohorts. In the gross anatomy laboratory course, every 20 students gets a single cadaver to practice dissection in one semester. Owing to the limited number of cadavers each year, these students treasured the opportunity to operate with their own hands, and generally were grateful to cadavers and their families [22]. They also tried their best to learn and hoped that they would pass qualifying exams to become a physician, who possesses high social status in Taiwan. The age of participants ranged $20 \sim 27$ (mean \pm
SD: $20.81 \pm 1.10$ ) years. In total, 46 participants had religious beliefs, and 68 of them had had an experience of the death of a friend or family member.

We collected data in their third year when they took the gross anatomy laboratory course; however, these students had gradually received teaching about gratitude toward 'silent mentors' since the start of their year two curriculum in medical school. Their lecturers had instructed them to think of the contribution of 'silent mentors' and the knowledge that they would gain from the 'silent mentors'. During the gross anatomy laboratory course, students spent 8 hours a week in a dissection room for 18 weeks. They spent an extra 8 hours reviewing body structures in the dissection room before the midterm and final exams.

\section{Our Intervention}

We instituted a series of activities to accompany our gross anatomy laboratory course at Taipei Medical University since 2010. Teachers and students conduct faceto-face interviews with the family members and/or friends of body donors at their houses or in a meeting room at the university. Students can then practically understand that the body donor on which they will work is not just a teaching instrument in a laboratory but was previously a living person. In the interview, students asked many questions about what kind of person the donor was, what he or she liked or hated, and why she or he decided to be a body donor. Through the interview, students could also understand that the family members and/or friends of the body donor would expect them to become excellent medical practitioners. After interviews of all groups, the Department of Anatomy and Cell Biology organizes an initiation ceremony in September at the beginning of the gross anatomy laboratory course with medical students, faculty members, and some relatives of the body donors in an auditorium at the university. The ceremony is carried out according to general Taiwanese rituals, without religious features, and cadavers are not exposed on-site. Students show their appreciation to the relatives, and promise that they will do their best to be good medical doctors in the future. In Taiwan, students and faculty of medical schools often call a body donor a 'silent mentor', who teaches students through donating his or her body. Therefore, students are learning with a teacher who does not speak. We expected that our intervention might substitute students'

Table 3 Research questions and hypotheses of the present study

\begin{tabular}{lll}
\hline No. & Research question & Hypothesis \\
\hline $\begin{array}{l}\text { 1. } \\
\text { emp activities such as family interviews and an initiation ceremony that }\end{array}$ & $\begin{array}{l}\text { Activities guiding students to have thankful thinking towards the } \\
\text { 'silent mentors' can increase students' high-level emotions. }\end{array}$ \\
2. & Do high-level emotions function to decrease students' negative emotions? & High-level emotions can decrease students' negative emotions. \\
\hline
\end{tabular}


negative emotions or psychological impacts with respect for the 'silent mentors'. On the other hand, based on feedback from our past interviews with students, an initiation ceremony can possibly fortify students' serious attitudes towards the gross anatomy laboratory course, as well as foster a thankful attitude to the body donors and their relatives.

\section{Measures}

Emotional Reactions Towards Cadavers Scale (ERTCS)

Based on students' descriptions after the first time they saw a cadaver and their emotional reactions observed by leaders of the Gross Anatomy Laboratory course, our research team listed 25 main emotions to measure students' emotional reactions after they see a cadaver. Items of the ERTCS are rated from 1 (strongly disagree) to 7 (strongly agree) on a Likert scale [22].

In order to categorize major emotional changes of students and validate the ERCTS, we conducted a pilot study that consisted of other 96 male and 62 female medical students $(n=158)$ at Taipei Medical University in 2015. Their ages ranged $19 \sim 30(20.97 \pm 1.53)$ years. The validity and reliability of the ERCTS are given in the 'Results' section.

\section{Self-Perceived Physical and Mental Condition and Relationship with their Families}

We asked participants to report their self-perceived physical and mental conditions, ranging from 1 (very good) to 5 (very tired). We also asked participants to report their relationships with their families, ranging from 1 (very good) to 5 (very bad).

\section{Experience of the Death of a Family Member or Friend and Demographic Variables}

We employed a yes/no question to ask participants whether they had had any experience of the death of a family member or friend. In addition, we collected participants' basic demographic variables including age, gender, and religious beliefs.

\section{Procedures}

\section{Research Procedures}

In their third year in medical school, participants filled out a measure of emotions and self-perceived physical and mental conditions three days before the 'silent mentor' initiation ceremony [time 1 (T1), on September 21, 2016]. They again filled out the emotional measurement form after the ceremony (T2, on October 3, 2016). To evaluate changes at the end of the semester, they also filled out the emotional measurement form at the end of the semester (T3, on January 13, 2017). A timeline of family interviews, initiation ceremony, and three-wave data collection is shown in Fig. 1.

\section{Ceremony Procedures}

Participants in the initiation ceremony included family members and friends of the 'silent mentors', all of the third-year medical students, and faculty members of the Department of Anatomy and Cell Biology. In the ceremony, each group of students was told about the silent mentors' past conduct and deeds to commemorate and appreciate them, and a wreath was laid.

\section{Data Processing}

We first conducted descriptive statistics including mean and standard deviation (SD) to show the distribution of the three emotions at different time points. Then we performed a repeated-measures analysis of variance (ANOVA) to examine whether the levels of the three emotions changed with time [36]. Finally, two hierarchical regression analyses were used to examine whether participants' high-level emotions at T2 and T3 could respectively predict their negative emotions at T2 and T3 when the participants' gender, religious beliefs, experience of the death of another, high-level emotions, and negative emotions at T1 were controlled for.

\section{Ethics}

The present study received ethical approval from the TMU-Joint Institutional Review Board (no. N201602066), and all methods were performed in accordance with relevant guidelines and regulations. Students volunteered to participate in the study, and informed consent was obtained from all participants. To avoid students' concerns about filling out the questionnaires, the instructions of the questionnaires stated that the results would not influence their scores, and they only needed to provide their student ID rather than their names to decrease their uncertainty.

\section{Results}

Prior to illustrating the main findings, the structure and consistency of the ERTCS are shown using data from our previous study [22]. To validate the ERTCS, we conducted an exploratory factor analysis, with the method of maximum likelihood factoring. The Kaiser-MeyerOlkin measure of sampling adequacy (0.86) and Bartlett's test of sphericity test $\left[\chi^{2}(300)=2140.96, p<0.001\right]$ for the correlation matrix showed that these data were suitable for a factor analysis [37]. The criterion of a eigenvalue $>1$ and Cattell's scree plot [38] were both considered to decide the number of axes, and the rotation method of promax was adopted as these emotions should be theoretically inter-correlated [39]. We found that a three-factor structure was the most meaningful result (Table 4), and $49.47 \%$ of the total variance was accounted for by the three factors, namely negative emotions (e.g., fear, being terrified, and being spooked, 
$23.40 \%$ ), high-level emotions (e.g., respect, reverence, and gratitude, $20.14 \%$ ), and excited emotions (e.g., full expectations, happiness, and curiosity, $5.93 \%)$. As for reliability, all of the 25 items showed high consistency (Cronbach's $\alpha=0.85$ ) [40]. Deleting any single item did not considerably elevate the consistency. Cronbach's $\alpha$ values of the three factors were $0.88,0.90$, and 0.84 , respectively. The ERCTS showed satisfactory validity and reliability. Thus, we further explored changes in these three emotions at the three time points.

Descriptive statistics are listed in Table 5, and emotional changes and significance levels are shown in Fig. 2. In general, participants reported high values for highlevel emotions, moderate values for excited emotions, and low values for negative emotions.

We conducted a repeated-measures ANOVA to examine whether the three types of emotions changed with time. The analysis observed significant within-subject effects on negative emotions $[\mathrm{F}(2,160)=22.62, p<0.001]$, and post-hoc comparisons with the Bonferroni correction revealed significant differences among T1 (3.00 \pm $0.89)$ contrasted with T2 $(2.54 \pm 0.91, p<0.001)$, T2 contrasted with T3 $(2.36 \pm 0.90, p<0.01)$, and T1 contrasted with T3 $(p<0.001)$. In addition, significant withinsubject effects on high-level emotions were observed $[\mathrm{F}(2,160)=6.05, \quad p<0.01]$, and post-hoc comparisons with the Bonferroni correction revealed significant differences among T1 $(5.68 \pm 0.83)$ contrasted with T2 $(5.95 \pm 0.81, p<0.05)$ and T1 contrasted with T3 $(5.99 \pm$ $0.95, p<0.01)$. Also, a significant within-subject effects on excited emotions was found $[\mathrm{F}(2,103)=15.96, p=$ $0.001)]$, and post-hoc comparisons with the Bonferroni correction revealed significant differences among $\mathrm{T} 1$ $(4.39 \pm 1.04)$ contrasted with T2 $(5.02 \pm 1.02, p<0.001)$, and $\mathrm{T} 1$ contrasted with $\mathrm{T} 3(4.77 \pm 1.20, p<0.001)$. Results showed that participants had fewer negative emotions after they participated in the ceremony (T1 vs. T2, $p<0.001$ ), and even fewer negative emotions at the end of the semester (T2 vs. T3, $p<0.01$ ). Participants had significantly more high-level emotions after they participated in the ceremony (T1 vs. T2, $p<0.05$ ), but with no significant change at the end of the semester (T2 vs. T3, $p>0.05)$. Participants had significantly more excited emotions after they participated in the ceremony (T1 vs. T2, $p<0.001$ ), and an insignificant drop in their excited emotions at the end of the semester (T2 vs. T3, $p>$ 0.05). In short, after the ceremony, participants had fewer negative emotions, more high-level emotions, and more excited emotions. At the end of the semester, their negative emotions continued to drop, high-level emotions remained high, and excited emotions had slightly rebounded.

Last, we conducted two hierarchical regression analyses to examine whether participants' high-level emotions at $\mathrm{T} 2$ and $\mathrm{T} 3$ would respectively predict their negative emotions at T2 and T3 when participants' highlevel emotions and negative emotions at $\mathrm{T} 1$ were controlled for. Results are shown in Tables 6 and 7.

In both analyses, at level 1, we controlled for confounding variables, including gender, having religious beliefs, self-perceived physical and mental conditions, and having had experienced the death of a family member or friend. At level 2, we controlled for participants' negative emotions and high-level emotions at T1. At level 3, we regressed participants' high-level emotions at $\mathrm{T} 2$ and T3 on their negative emotions at T2 and T3, respectively. Results showed that participants' high-level emotions at both T2 and T3 significantly and negatively predicted their negative emotions at T2 and T3.

\section{Discussion}

The present study deployed three different statistical techniques, and sequentially found three important results. First, we distinguished high-level emotions and negative emotions, which respectively were our explanatory variable and response variable, from excited emotions with an exploratory factor analysis. Second, stable changes at three time points-rises in high-level emotions and declines in negative emotions-achieved significance with the repeated-measures ANOVA. Third, after controlling for impacts of external factors, negative emotions could be predicted not only by their previous value, but also by high-level emotions at the same time point according to the hierarchical regression analyses.

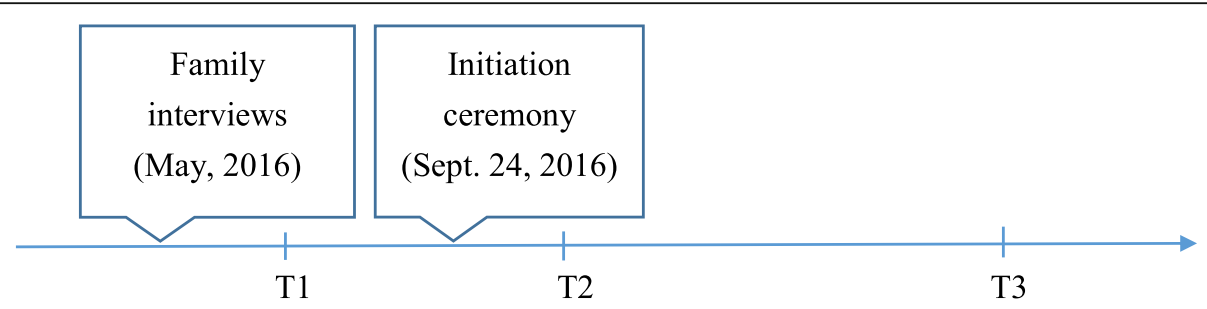

(Sept. 21, 2016) (Oct. 3, 2016)

(Jan. 13, 2017)

Fig. 1 Timeline of family interviews, initiation ceremony, and three-wave data collection. 
Table 4 Factor structures of the Emotional Reactions Towards Cadaver Scale

\begin{tabular}{|c|c|c|c|}
\hline & \multicolumn{3}{|l|}{ Factor } \\
\hline & Negative emotions & High-level emotions & Excited emotions \\
\hline Fear & 0.79 & -0.11 & -0.08 \\
\hline Being terrified & 0.76 & -0.14 & 0.09 \\
\hline Being spooked & 0.74 & -0.23 & -0.01 \\
\hline Shock & 0.68 & 0.03 & 0.07 \\
\hline Pain & 0.67 & -0.18 & -0.02 \\
\hline Feeling low & 0.63 & -0.21 & -0.14 \\
\hline Being unlucky & 0.59 & -0.37 & -0.04 \\
\hline Avoidance as a taboo & 0.59 & -0.19 & 0.04 \\
\hline Feeling like escaping & 0.59 & -0.24 & -0.34 \\
\hline Grief & 0.50 & 0.20 & 0.18 \\
\hline Nervousness & 0.46 & 0.27 & 0.26 \\
\hline Too sympathetic to bear & 0.39 & 0.26 & 0.24 \\
\hline Doubt (to future processes on a cadaver) & 0.35 & 0.15 & 0.32 \\
\hline Disappointment (an inability to achieve others' expectations) & 0.35 & 0.06 & 0.07 \\
\hline Respect & -0.12 & 0.89 & 0.43 \\
\hline Cherishing & -0.08 & 0.88 & 0.50 \\
\hline Gratitude & -0.18 & 0.85 & 0.57 \\
\hline Admiration & -0.10 & 0.82 & 0.48 \\
\hline Humility & -0.04 & 0.77 & 0.34 \\
\hline Peace & -0.40 & 0.48 & 0.36 \\
\hline Full expectation & -0.03 & 0.51 & 0.80 \\
\hline Happiness & -0.01 & 0.51 & 0.77 \\
\hline Curiosity & 0.06 & 0.36 & 0.72 \\
\hline Familiarity (with the 'silent mentor') & -0.02 & 0.56 & 0.67 \\
\hline Excitement & 0.10 & 0.19 & 0.62 \\
\hline Eigenvalue & 5.85 & 5.04 & 4.48 \\
\hline$\%$ of variance explained & 23.40 & 20.14 & 5.93 \\
\hline Cronbach's a-coefficient & 0.88 & 0.90 & 0.84 \\
\hline
\end{tabular}

Table 5 Descriptive statistics and repeated-measures analysis of variance (ANOVA) of the three emotions at three time points and self-perceived conditions

\begin{tabular}{|c|c|c|c|c|c|c|c|c|c|}
\hline & \multicolumn{2}{|l|}{ Time 1} & \multicolumn{2}{|c|}{ Time 2} & \multicolumn{2}{|l|}{ Time 3} & \multicolumn{3}{|c|}{ Repeated-measures ANOVA } \\
\hline & Mean & SD & Mean & SD & Mean & SD & F & $d f$ & $p$ \\
\hline Negative emotions & 3.00 & 0.89 & 2.54 & 0.91 & 2.36 & 0.90 & 22.62 & 2,160 & $<0.001$ \\
\hline High-level emotions & 5.68 & 0.83 & 5.95 & 0.81 & 5.99 & 0.95 & 6.05 & 2,160 & $<0.001$ \\
\hline Excited emotions & 4.39 & 1.04 & 5.02 & 1.02 & 4.77 & 1.20 & 15.96 & 2,160 & $<0.001$ \\
\hline Self-perceived condition & 1.67 & 0.71 & & & & & & & \\
\hline
\end{tabular}

Emotions were rated by the Emotional Reactions Towards Cadavers Scale with a Likert-type scale from 1 (strongly disagree) to 7 (strongly agree) SD standard deviation 


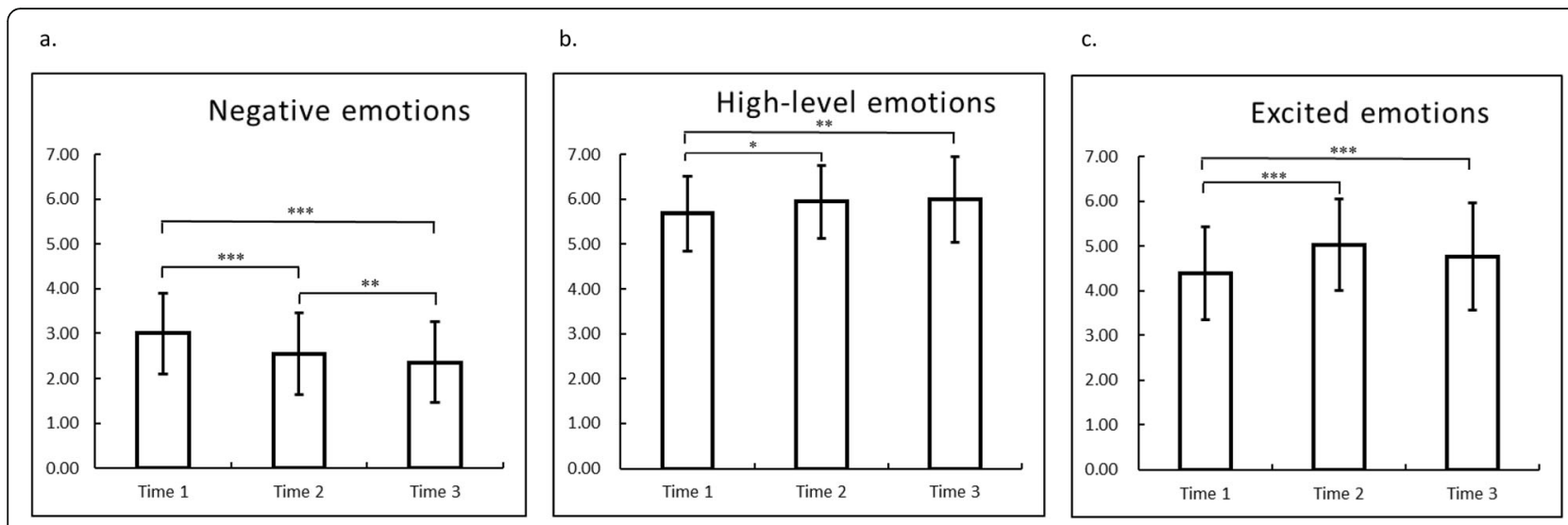

Fig. 2 Changes and significance levels of the three emotions (a. negative emotions; $\mathbf{b}$. high-level emotions; $\mathbf{c}$. excited emotions) at three time points. ${ }^{*} p<0.05,{ }^{* *} p<0.01,{ }^{* * *} p<0.001$.

The consideration of arranging our results in these three steps did not come from statistical concerns, but relied on a post-positivism epistemological standpoint, which determined our study design, methods, and data analyses. In this regard, we provide our discussion of these results as follows.

Although the literature on coping strategies, whether coming from educational interventions or from students' adoption during the gross anatomy laboratory course, crucial emotional reactions still remain under- elaborated, and related factors undifferentiated [5, 7, 8, $12,13,15]$. We elaborated this problem with the ERTCS by distinguishing three different emotions-negative, high-level, and excited emotions-and then established a relationship between high-level emotions and negative emotions. Scarcely mentioned in the previous literature $[4-7,9,13-15,17-21]$, our study showed that high-level emotions were relatively high and negative emotions were relatively low. High-level emotions represent an achievement of our educational intervention, and were

Table 6 Hierarchical regression analyses of high-level emotions to negative emotions at T2

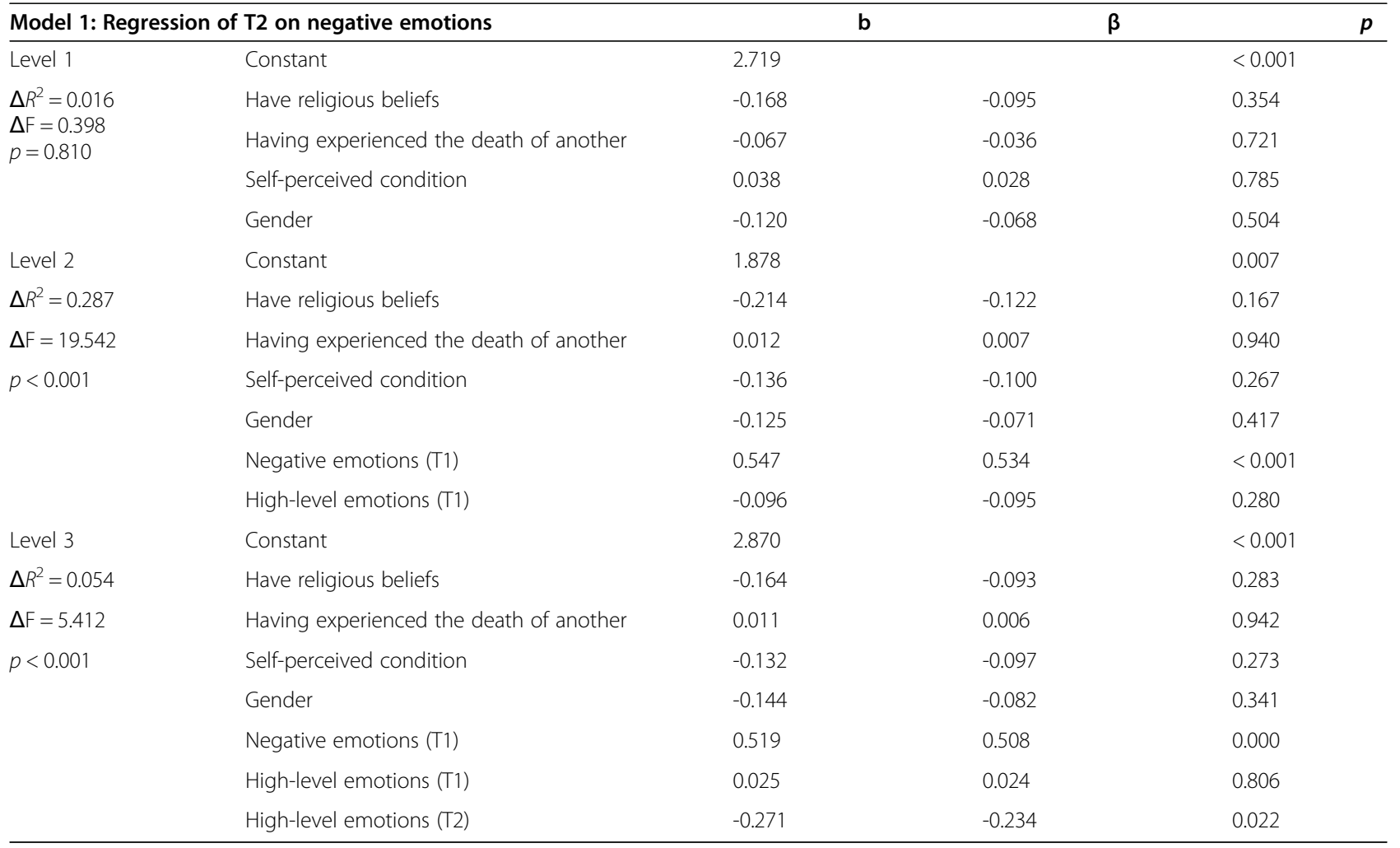


Table 7 Hierarchical regression analyses of high-level emotions to negative emotions at T3

\begin{tabular}{|c|c|c|c|c|}
\hline \multicolumn{2}{|c|}{ Model 2: Regression of T3 on negative emotions } & \multirow{2}{*}{$\begin{array}{l}\mathrm{b} \\
2.556\end{array}$} & \multirow[t]{2}{*}{$\beta$} & \multirow{2}{*}{$\begin{array}{l}p \\
<0.00\end{array}$} \\
\hline Level 1 & Constant & & & \\
\hline \multirow[t]{4}{*}{$\Delta R^{2}=0.007 \Delta \mathrm{F}=0.163 p=0.957$} & Have religious beliefs & -0.043 & -0.025 & 0.816 \\
\hline & Having experienced the death of another & -0.051 & -0.028 & 0.793 \\
\hline & Self-perceived condition & -0.068 & -0.058 & 0.595 \\
\hline & Gender & -0.060 & -0.035 & 0.748 \\
\hline Level 2 & Constant & 1.902 & & 0.008 \\
\hline$\Delta R^{2}=0.137$ & Have religious beliefs & -0.062 & -0.037 & 0.718 \\
\hline$\Delta \mathrm{F}=6.889$ & Having experienced the death of another & -0.021 & -0.012 & 0.906 \\
\hline \multirow[t]{4}{*}{$p=0.002$} & Self-perceived condition & -0.157 & -0.134 & 0.203 \\
\hline & Gender & -0.040 & -0.023 & 0.821 \\
\hline & Negative emotions (T1) & 0.365 & 0.369 & 0.001 \\
\hline & High-level emotions (T1) & -0.058 & -0.062 & 0.539 \\
\hline Level 3 & Constant & 3.487 & & $<0.001$ \\
\hline$\Delta R^{2}=0.113$ & Have religious beliefs & 0.030 & 0.018 & 0.856 \\
\hline$\Delta \mathrm{F}=12.963$ & Having experienced the death of another & 0.013 & 0.007 & 0.938 \\
\hline \multirow[t]{5}{*}{$p=0.001$} & Self-perceived condition & -0.269 & -0.229 & 0.026 \\
\hline & Gender & -0.045 & -0.026 & 0.787 \\
\hline & Negative emotions (T1) & 0.347 & 0.351 & $<0.001$ \\
\hline & High-level emotions (T1) & 0.098 & 0.104 & 0.323 \\
\hline & High-level emotions (T3) & -0.382 & -0.390 & 0.001 \\
\hline
\end{tabular}

the main effect of the initiation ceremony and family interviews, while negative emotions were a crucial and refined indicator of medical students' emotional reactions toward cadavers during the course. With the help of differentiation, we not only viewed high-level emotions as a predictive variable and negative emotions as a dependent variable by separating them from excited emotions, which could be a confounding factor, but also analytically considered both the diachronic relationship within high-level emotions or negative emotions and the synchronic relationship between the 2 types of emotion. Only when the relationship among different emotional reactions were elaborated could we researchers scrutinize the 'coping strategies' designed by educators. In other words, we found that the educational intervention, both the initiation ceremony and the family interviews, could raise students' high-level emotions and decrease students' negative emotions.

Based on this primary finding, we first demonstrated the question of how students' high-level emotions can decrease their negative emotions after controlling for demographic factors. Within the trends of high-level emotions continuing to rise and negative emotions continuing to drop from $\mathrm{T} 1$ to $\mathrm{T} 2$ and $\mathrm{T} 3$, the three-step hierarchical or sequential regression analyses showed three crucial points worth discussing, which might echo both the broaden-and-build theory in positive psychology in general and the state-gratitude as a positive psychological motive in particular.

First, external factors, represented by variables we controlled for, such as gender, religion, experience of a death of another, and self-perceived conditions, had no significant effects on students' negative emotions at T2 or T3. Although our findings did not exclusively reject previous research about different emotional reactions in terms of gender or religious factors [14, 17, 20, 31], those external factors actually had no influences when the diachronic and synchronic relationships between different emotional reactions were considered. This basic finding enabled us at an early point to shift our focus from external factors to internal factors, indicating a series of psychological mechanisms that needed to be explored. With the help of the broaden-and-build theory, we, however, traced the psychological process neither in medical student's individual coping strategies [1, $2,5,7,8,13-15]$, nor in medical educators' interventional strategies [10-12], but directly in distinguishing student's emotional reactions in order to find a solution within $[16,22]$. On the one hand, after sorting out highlevel emotions, we found that many of them, such as 'respect, gratitude, admiration, and humility', could be recognized as state-gratitude after the initiation ceremony and the family interview; on the other hand, these 
emotions might not only 'broaden' medical student's compassion for donor's family, but also 'build' their own confidence in facing cadavers in the present and preparedness for death in the future.

Second, both models 1 and 2 in the hierarchical regression analysis showed that high-level emotions might have a synchronic impact on negative emotions. Results indicated an elaborate relationship between consistently rising high-level emotions and consistently dropping negative emotions. More important to us was the fact that the impact of highlevel emotions on negative emotions became stronger ( $b=-0.271$ in model $1 ; b=-0.382$ in model 2 ), while the impact of previous negative emotions on negative emotions became weaker $(b=0.591$ in model $1 ; b=$ 0.347 in model 2). This advanced finding supports our research argument: although there is much research on mitigating negative emotions and coping strategies, medical students' negative emotions gradually decreased, which might have been a foreseeable consequence of students' adaptation [9-11, 18, 20]. On the contrary, the often-neglected factor, actively and positively reducing students' negative emotions, should come from another rising factor which was the result of the educational intervention combined with students' perceptions. In our research findings, consistently rising high-level emotions played a role as an active, positive factor, and filled the vacuum in research on students' negative emotions. Interestingly, our findings confirmed previous research and somehow extended their conclusions that naming the body donor helped students overcome their uneasiness of seeing a cadaver [16]. In fact, naming and related activities-remembering the donor's personality, thinking of the donor's life, or approaching the donor's relatives-belonged to events that could raise student's high-level emotion as gratitude. In addition, this also reconfirmed and echoed our previous research that rituals with a humanistic design decrease negative emotions towards cadavers during the learning process [22].

Third, educational interventions such as the initiation ceremony and family interviews might have two different mechanisms mitigating students' negative emotions. In a directly influencing mechanism, we found that students' negative emotions decreased after the initiation ceremony and family interviews. Students definitely adapted to the uncomfortable feelings toward the cadaver dissection; however, our previous study indicated that rituals or activities with a humanistic design, such as the initiation ceremony, could significantly facilitate student's adaptation and efficiently mitigate their uneasiness [22]. However, there might be another yet undetected way, an indirectly influencing mechanism, of mitigation. In this study, we elaborated that on the one hand, high-level emotions increased after the initiation ceremony and family interviews, and high-level emotions also caused a mitigating impact on negative emotions. Only after we established relationships among the educational intervention, high-level emotions, and negative emotions was the indirectly influencing mechanism obviously revealed. This underlying relationship is not only a kind of moral feelings that cultivated student's knowledge of life education, but also a psychological interaction between highlevel emotions and negative emotions, which was coined with the term of either 'reframing emotion' or 'undoing effect' in positive psychology $[23,24,26,27]$. In this regard, our finding that high-level emotions can mitigate negative emotions could be interpreted as a kind of 'reframing negative emotions with high-level emotions' or 'undoing negative emotions with high-level emotions' through state gratitude as a positive psychological motive.

In summary, the present study demonstrated that (1) educational interventions, such as the initiation ceremony and family interviews, can significantly increase high-level emotions and decrease negative emotions; and (2) high-level emotions can significantly decrease negative emotions under a condition in which external factors are controlled. These two related findings indicate an interesting fact that after differentiating emotions into high-level, excited, and negative emotions, we found an internal way of mitigating medical students' negative emotions during a gross anatomy laboratory course. Trying to elaborate the internal causality between high-level emotions and negative emotions has become a new issue worth noting, while past research usually focused on coping strategies, whether personal or institutional ones $[1,2,5,7,8,10-15]$.

Furthermore, high-level emotions based on the ERTCS (see Table 4) echo the concept of gratitude, which had a buffering effect of decreasing negative emotions. We could extend this confirmative finding in advance with the broaden-and-build theory in positive psychology: when high-level emotions show various similarities to state gratitude, they could act as a positive psychological motive during the cadaver dissection course, through both 'broaden' and 'build' mechanisms, to mitigate students' negative emotional reactions. This mitigating interaction of high-level emotions on negative emotions performed as much as the 'reframing emotion' or the 'undoing effect' on other positive emotions [23, 24, 26, 27].

\section{Limitations}

From a research design viewpoint, we should have utilized experimental and control groups to examine the intervention effects; however, students were willing to 
participate in these activities because of the value and meaning. Thus, a hierarchical regression was used to control for unnecessary influences to compensate for this study design issue. We believe that medical education research cannot always rely on mainstream approaches deployed in a randomized controlled trial; and yet we can gain valuable insights by combining experimental or educational interventions with quantitative or qualitative data. Randomized controlled trials are not the gold-standard methodological approach to medical education research in the way that such trials are in medical science. In this sense, we suggest triangulation which can ensure both validity and reliability.

From comparisons between Taiwan and other countries, medical students in our research sample reflected a lower scope of negative emotions, which implies that there was seemingly no dramatic mitigation of all kinds of negative emotions as has occurred in the contexts of other research. However, to what extent high-level emotions can mitigate negative emotions under paired research conducted in different countries with their own cultural contexts would be an interesting question to explore. Therefore, we suggest that comparisons between Taiwan and other countries be conducted in the future.

The last limitation is that we did not perform a power calculation to identify the adequate sample size prior to executing the study. Considering the cadaver dissection as a constant course, we suggested establishing longitudinal data enabling researchers to testify the long-term effects of positive psychological motives. This will enrich research into cadaver dissection courses with the current situation where a cross-sectional study is the most commonly seen method.

\section{Conclusions}

Combining dissection courses with medical humanities is helpful for students coping with their negative emotions during a gross anatomy laboratory course. Our research findings show that high-level emotions significantly mitigated negative emotions indicating practical implications in medical education by combining body dissection with medical humanities. As suggested by our study, direct deploy biographical information or life story of a 'silent mentor' should be used rather than indirectly developing students' coping strategies. Therefore, holding curricular activities like family interviews or ceremonies is a good strategy to alleviate students' negative emotions, and they also bring other positive learning effects according to our past study [22]. We believe that the implications of our study will both facilitate instructors' practice and improve students' learning effectiveness in gross anatomy laboratory courses.

\section{Abbreviations}

ANOVA: Analysis of variance; ERCTS: Emotional Reactions Towards Cadaver Scale; SD: Standard deviation

\section{Acknowledgements}

The authors would like to thank the support of English editing services from the Office of Research and Development, Taipei Medical University.

\section{Authors' contributions}

RJC and DYH conceived of the present idea. RJC acquired the data. PFT and DYH developed the theory, performed the computations, and verified the analytical methods. All authors discussed the results and approved the final manuscript.

\section{Funding}

RJC received funding support from the Ministry of Science and Technology, Taiwan under grant MOST105-2511-S-038-006-MY2, and Taipei Medical University under grant TMU101-AE1-B50. PFT received funding support from Ministry of Science and Technology, Taiwan under grant MOST108-2410-H038-012-MY2.

\section{Availability of data and materials}

The datasets generated and analyzed during the current study are not publicly available due to protection of the privacy of subjects, but are available from the corresponding author upon reasonable request.

\section{Declarations}

Ethics approval and consent to participate

The study received ethics approval from the Joint Institutional Review Board of Taipei Medical University (April 26, 2016, no. N201602066). Informed consent was obtained from participating students. The donor/next of kin of cadavers signed agreements when they decided to donate.

\section{Consent for publication}

Not applicable.

\section{Competing interests}

The authors declare they have no conflicts of interest.

\section{Author details}

'Department of Anatomy and Cell Biology, School of Medicine, College of Medicine, Taipei Medical University, Taipei, Taiwan. ${ }^{2}$ Graduate Institute of Humanities in Medicine, College of Humanities and Social Sciences, Taipei Medical University, Taipei, Taiwan. ${ }^{3}$ Section of Liberal Arts, Center for General Education, Taipei Medical University, 250 Wu-Xing St., Xinyi District, 11031

Taipei, Taiwan

Received: 26 January 2021 Accepted: 31 August 2021

Published online: 11 September 2021

\section{References}

1. GetachewD. Reaction of medical students to experiences in dissection room. Ethiop J Health Sci. 2014;24:337-42.

2. BobMH, PopescuCA, ArmeanMS, SuciuSM, BuzoianuAD. Ethical views, attitudes and reactions of Romanian medical students to the dissecting room. Rev Med Chir Soc Med Nat lasi. 2014;118:1078-85.

3. BernhardtV, RothkötterHJ, KastenE. Psychological stress in first year medical students in response to the dissection of a human corpse. GMS Z Med Ausbild. 2012;29:Doc12.

4. DempsterM, BlackA, McCorryN, WilsonD. Appraisal and consequences of cadaver dissection. Med Educ Online. 2006;11:4592.

5. BatainehZM, HijaziTA, HijlehMFA. Attitudes and reactions of Jordanian medical students to the dissecting room. Surg Radiol Anat. 2006;28:416-21.

6. SaylamC, CoskunolH. Orientation lesson in anatomy education. Surg Radiol Anat. 2005;27:74-7.

7. HouwinkAP, KurupAN, KollarsJP, KollarsCAK, CarmichaelSW, PawlinaW. Help of third-year medical students decreases first-year medical students' negative psychological reactions on the first day of gross anatomy dissection. Clin Anat. 2004;17:328-33. 
8. Sándorl, BirkásE, GyorffyZ. The effects of dissection-room experiences and related coping strategies among Hungarian medical students. BMC Med Educ. 2015;15:73.

9. Arráez-AybarLA, Castaño-ColladoG, Casado-MoralesMI. Dissection as a modulator of emotional attitudes and reactions of future health professionals. Med Educ. 2008;42:563-71.

10. Arráez-AybarLA, Casado-MoralesMl, Castaño-ColladoG. Anxiety and dissection of the human cadaver: An unsolvable relationship? Anat Rec Part B New Anat. 2004;279:16-23.

11. CasadoMl, CastañoG, Arráez-AybarLA. Audiovisual material as educational innovation strategy to reduce anxiety response in students of human anatomy. Adv Heal Sci Educ. 2012;17:431-40.

12. DosaniF, NeubergerL. Anatomy and humanity: Examining the effects of a short documentary film and first anatomy laboratory experience on medical students. Anat Sci Educ. 2016:9:28-39.

13. KotzéSH, MoleCG. Talking about death: Implementing peer discussion as a coping mechanism to overcome fears about dissection, death, and dying. Anat Sci Educ. 2013;6:191-8.

14. LamdinR, WellerJ, KerseN. Orientation to dissection: Assisting students through the transition. Clin Anat. 2012;25:235-40.

15. TsengWT, LinYP. "Detached concern" of medical students in a cadaver dissection course: A phenomenological study. Anat Sci Educ. 2016;9:265-71.

16. WilliamsAD, GreenwaldEE, SoricelliRL, DePaceDM. Medical students' reactions to anatomic dissection and the phenomenon of cadaver naming. Anat Sci Educ. 2014;7:169-80

17. QuinceTA, BarclaySIG, SpearM, ParkerRA, WoodDF. Student attitudes toward cadaveric dissection at a UK medical school. Anat Sci Educ. 2011;4:200-7.

18. CahillKC, EttarhRR. Attitudes to anatomy dissection in an Irish medical school. Clin Anat. 2009;22:386-91.

19. Qamark, OsamaM. Role of dissection in light of students' perceptions. J Pak Med Assoc. 2014;64:1021-4.

20. SnellingJ, SahaiA, EllisH. Attitudes of medical and dental students to dissection. Clin Anat. 2003;16:165-72.

21. BatiAH, OzerMA, GovsaF, PinarY. Anxiety of first cadaver demonstration in medical, dentistry and pharmacy faculty students. Surg Radiol Anat. 2013;35: 419-26.

22. ChiouRJ, TsaiPF, HanDY. Effects of a "silent mentor" initiation ceremony and dissection on medical students' humanity and learning. BMC Res Notes. 2017;10.

23. FredricksonBL. Gratitude, like other positive emotions, broadens and builds. In: The Psychology of Gratitude. New York: Oxford University Press; 2004 $145-66$.

24. McCulloughME, EmmonsRA, KilpatrickSD, LarsonDB. Is gratitude a moral affect? Psychol Bull. 2001;127:17.

25. FredricksonBL. The broaden-and-build theory of positive emotions. Philos Trans R Soc B Biol Sci. 2004;359:1367-78.

26. FredricksonBL, MancusoRA, BraniganC, TugadeMM. The undoing effect of positive emotions. Motiv Emot. 2000;24:237-58.

27. LambertNM, FinchamFD, StillmanTF. Gratitude and depressive symptoms: The role of positive reframing and positive emotion. Cogn Emot. 2012;26: 615-33.

28. SimonP. An exploratory study about the buffering effect of gratitude on the relationship between neuroticism and anhedonic depression. Psychol Stud (Mysore). 2016;61:340-7.

29. RilloAG, Martínez-CarrilloBE, Castillo-CardielJA, Rementería-SalinasJM. Constructivism: An interpretation from medical education. IOSR J Res Method Educ. 2020;10:1-12.

30. WoodAM, MaltbyJ, StewartN, LinleyPA, JosephS. A social-cognitive model of trait and state levels of gratitude. Emotion. 2008;8:281.

31. BöckersA, BaaderC, FassnachtUK, ÖchsnerW, BöckersTM. Reduction of mental distress in the dissection course by introducing the body donor experience through anatomical demonstrations of organ systems. Anat Sci Educ. 2012:5:321-9.

32. PachecoJPG, GiacominHT, TamWW, RibeiroTB, ArabC, BezerralM, et al. Mental health problems among medical students in Brazil: A systematic review and meta-analysis. Brazilian J Psychiatry. 2017:39:369-78.

33. FrajermanA, MorvanY, KrebsMO, GorwoodP, ChaumetteB. Burnout in medical students before residency: A systematic review and meta-analysis. Eur Psychiatry. 2019;55:36-42.

34. PhillipsDC \& BurbulesNC. Postpositivism and Educational Research. Lanham \& Boulder: Rowman \& Littlefield Publishers; 2000.
35. LaudanL. Beyond Positivism and Relativism: Theory, Method, and Evidence Boulder, CO: Westview Press; 1996.

36. SinghV, RanaRK, SinghalR. Analysis of repeated measurement data in the clinical trials. J Ayurveda Integr Med. 2013:4:77-81.

37. KaiserHF, RiceJ. Little Jiffy, Mark IV. Educ Psychol Meas. 1974;34:111-7.

38. CattellRB. The scree test for the number of factors. Multivariate Behav Res. 1966;1:245-76.

39. HendricksonAE, WhitePO. Promax: A quick method for rotation to oblique simple structure. Br J Stat Psychol. 1964:17:65-70.

40. TaberkS. The use of Cronbach's alpha when developing and reporting research instruments in science education. Res Sci Educ. 2018:48:1273-96.

\section{Publisher's Note}

Springer Nature remains neutral with regard to jurisdictional claims in published maps and institutional affiliations.
Ready to submit your research? Choose BMC and benefit from:

- fast, convenient online submission

- thorough peer review by experienced researchers in your field

- rapid publication on acceptance

- support for research data, including large and complex data types

- gold Open Access which fosters wider collaboration and increased citations

- maximum visibility for your research: over $100 \mathrm{M}$ website views per year

At BMC, research is always in progress.

Learn more biomedcentral.com/submissions 